Congr uence of Si egel nodul ar for ms and speci al val ues of thei $r$ standard zet a functions

\begin{tabular}{|l|l|}
\hline 著者 & KATSURADA Hi denor i \\
\hline $\begin{array}{l}\text { j our nal or } \\
\text { publ i cat i on } \mathrm{t} \mathrm{i} \mathrm{t} \mathrm{l} \mathrm{e}\end{array}$ & Nat henat i sche Zei t schr i ft \\
\hline vol une & 259 \\
\hline number & 1 \\
\hline page $\mathrm{r}$ ange & $97-111$ \\
\hline year & 2008 05 \\
\hline URL & ht t p: //hdl . handl e. net /10258/439 \\
\hline
\end{tabular}


Congr uence of Si egel nodul ar for ms and speci al val ues of thei $r$ standard zet a functions

\begin{tabular}{|l|l|}
\hline 著者 & KATSURADA Hi denor i \\
\hline $\begin{array}{l}\text { j our nal or } \\
\text { publ i cat i on } \mathrm{t} \mathrm{i} \mathrm{t} \mathrm{l} \mathrm{e}\end{array}$ & Nat henat i sche Zei t schr i ft \\
\hline vol une & 259 \\
\hline number & 1 \\
\hline page $\mathrm{r}$ ange & $97-111$ \\
\hline year & 2008 05 \\
\hline URL & ht t p: //hdl . handl e. net /10258/439 \\
\hline
\end{tabular}




\title{
Congruence of Siegel modular forms and special values of their standard zeta functions
}

Received: date / Revised: date

\begin{abstract}
In this paper, we consider the relationship between the congruence of cuspidal Hecke eigenforms with respect to $S p_{n}(\mathbf{Z})$ and the special values of their standard zeta functions. In particular, we propose a conjecture concerning the congruence between Saito-Kurokawa lifts and non-SaitoKurokawa lifts, and prove it under certain condition.
\end{abstract}

Mathematics Subject Classification (2000) 11F46, 11F33,11F67

\section{Introduction}

For a cuspidal Hecke eigenform $f$ of weight $k$ with respect to $S p_{n}(\mathbf{Z})$, let $L(f, s, \underline{\mathrm{St}})$ be the standard zeta function of $f$. Let $m$ be a positive integer such that $\rho(n) \leq m \leq k-n$ and $m \equiv n \bmod 2$, where $\rho(n)=3$, or 1 accorrding as $n \equiv 1 \bmod 4$ and $n \geq 5$, or not. Then the value $\frac{L(f, m, \underline{\mathrm{St}})}{<f, f>\pi}$

belongs to $\mathbf{Q}(f)$ if all the Fourier coefficients of $f$ belong to $\mathbf{Q}(f)$, where $\langle f, f\rangle$ is the Petersson product and $\mathbf{Q}(f)$ is the field over $\mathbf{Q}$ generated by all Hecke eigenvalues (cf. [3], [25]). In this paper, we consider the relation between these values and the congruence of Hecke eigenvalues of cusp forms. This type of problem was first considered by Doi and Hida [7] in terms of special values of Rankin-Selberg zeta functions, and by Hida [13] in terms of special values of standard zeta functions in the elliptic modular case. In [17]

Partially supported by Grant-in-Aid for Scientific Research C-17540003, JSPS

H. Katsurada

Muroran Institute of Technology, 27-1 Mizumoto Muroran, 050-8585, Japan

Tel.: +143-46-5804

Fax: +143-46-5804

E-mail: hidenori@mmm.muroran-it.ac.jp 
and [18], we also considered this problem in a different way from theirs. In Section 5, we give a generalization of our result in terms of the denominantor of special values of standard zeta functions in the Siegel modular form case (cf. Theorems 5.2 and 5.3). The main tool for proving our main results is the pullback formula for Siegel Eisenstein series due to Böcherer [2], [3], and Garrett [10], which we will review in Section 4. This formula has been already used to prove to algebraicity of the special values of the standard zeta functions stated above. However, to complete the proof of our main results, we have to consider the integrality of the Eisenstein series acted by a certain differential operators. We will discuss this integrality in Sections 2 and 3 . Furthermore, to formulate our main results reasonably, we have to consider a normalization of the standard zeta values because we have no normalization of Hecke eigenforms in case $n \geq 2$ unlike the elliptic modular case. We discuss this normalization of the standard zeta values in Section 5 . Furthermore, in Section 6, we propose a conjecture concerning the congruence between Saito-Kurokawa lifts and non-Saito-Kurokawa lifts, and prove it under certain condition. We note that this type of conjecture has been proposed by Harder [11], [12] under more general situation. We note that we can formulate this type of conjecture for congruence primes of the Ikeda lift, which we will discuss in a subsequent paper. We also note that we can give exact values of the standard zeta function by using our method (cf. [19]).

Notation. For a commutative ring $R$, we denote by $M_{m n}(R)$ the set of $(m, n)$-matrices with entries in $R$. In particular put $M_{n}(R)=M_{n n}(R)$. Here we understand $M_{m n}(R)$ the set of the empty matrix if $m=0$ or $n=0$. For an $(m, n)$-matrix $X$ and an $(m, m)$-matrix $A$, we write $A[X]={ }^{t} X A X$, where ${ }^{t} X$ denotes the transpose of $X$. Let $a$ be an element of $R$. Then for an element $X$ of $M_{m n}(R)$ we often use the same symbol $X$ to denote the coset $X \bmod a M_{m n}(R)$. Put $G L_{m}(R)=\left\{A \in M_{m}(R) \mid \operatorname{det} A \in R^{*}\right\}$, where $\operatorname{det} A$ denotes the determinant of a square matrix $A$, and $R^{*}$ denotes the unit group of $R$. Let $S_{n}(R)$ denote the set of symmetric matrices of degree $n$ with entries in $R$. Furthermore, for an integral domain $R$ of characteristic different from 2 , let $\mathcal{H}_{n}(R)$ denote the set of half-integral matrices of degree $n$ over $R$, that is, $\mathcal{H}_{n}(R)$ is the set of symmetric matrices of degree $n$ whose $(i, j)$-component belongs to $R$ or $\frac{1}{2} R$ according as $i=j$ or not. For a subset $S$ of $M_{n}(R)$ we denote by $S^{\times}$the subset of $S$ consisting of non-degenerate matrices. In particular, if $S$ is a subset of $S_{n}(\mathbf{R})$ with $\mathbf{R}$ the field of real numbers, we denote by $S_{>0}$ (resp. $S_{>0}$ ) the subset of $S$ consisting of positive definite (resp. semi-positive definite) matrices. Let $R^{\prime}$ be a subring of $R$. Two symmetric matrices $A$ and $A^{\prime}$ with entries in $R$ are called equivalent over $R^{\prime}$ with each other and write $A \widetilde{R^{\prime}} A^{\prime}$ if there is an element $X$ of $G L_{n}\left(R^{\prime}\right)$ such that $A^{\prime}=A[X]$. We also write $A \sim A^{\prime}$ if there is no fear of confusion. For square matrices $X$ and $Y$ we write $X \perp Y=\left(\begin{array}{cc}X & O \\ O & Y\end{array}\right)$. 


\section{Fourier coefficients of Siegel-Eisenstein series}

For a complex number $x$ put $\mathbf{e}(x)=\exp (2 \pi \sqrt{-1} x)$. Furthermore put $J_{n}=$ $\left(\begin{array}{cc}O_{n} & -1_{n} \\ 1_{n} & O_{n}\end{array}\right)$, where $1_{n}$ denotes the unit matrix of degree $n$. For a subring $K$ of $\mathbf{R}$ put

$$
G S p_{n}(K)^{+}=\left\{M \in G L_{2 n}(K) \mid J_{n}[M]=\kappa(M) J_{n} \text { with some } \kappa(M)>0\right\},
$$

and

$$
S p_{n}(K)=\left\{M \in G S p_{n}(K)^{+} \mid J_{n}[M]=J_{n}\right\} .
$$

Furthermore, put

$$
\Gamma^{(n)}=S p_{n}(\mathbf{Z})=\left\{M \in G L_{2 n}(\mathbf{Z}) \mid J_{n}[M]=J_{n}\right\} .
$$

Let $\mathbf{H}_{n}$ be Siegel's upper half-space. For each element $M=\left(\begin{array}{ll}A & B \\ C & D\end{array}\right) \in$ $G S p_{n}(\mathbf{R})^{+}$and $Z \in \mathbf{H}_{n}$ put

$$
M(Z)=(A Z+B)(C Z+D)^{-1}
$$

and

$$
j(M, Z)=\operatorname{det}(C Z+D) .
$$

Furthermore, for a function $f$ on $\mathbf{H}_{n}$ we define $\left.f\right|_{k} M$ as

$$
\left(\left.f\right|_{k} M\right)(Z)=\operatorname{det}(M)^{k / 2} j(M, Z)^{-k} f(M(Z)) .
$$

A function $f$ on $\mathbf{H}_{n}$ is called a $C^{\infty}$-modular form of weight $k$ with respect to $\Gamma^{(n)}$ if it satisfies the following conditions:

(i) $f$ is a $C^{\infty}$-function on $\mathbf{H}_{n}$;

(ii) $\left(\left.f\right|_{k} M\right)(Z)=f(Z)$ for any $M \in \Gamma^{(n)}$;

We call a $C^{\infty}$-modular form $f$ a holomorphic modular form if (i) $f$ is holomorphic on $\mathbf{H}_{n}$ :

(ii) if $n=1$, for any $\alpha>0, f(z)$ is bounded on the set $\{x+\sqrt{-1} y \mid y \geq \alpha\}$ for each $\alpha>0$.

We denote by $\mathfrak{M}_{k}\left(\Gamma^{(n)}\right)$ (resp. $\mathfrak{\Re}_{k}^{\infty}\left(\Gamma^{(n)}\right)$ the space of holomorphic (resp. $C^{\infty}$-) modular forms of weight $k$ with respect to $\Gamma^{(n)}$. For a modular form $f$ of weight $k$ with respect to $\Gamma^{(n)}$, let

$$
f(Z)=\sum_{A \in \mathcal{H}(\mathbf{Z})_{\geq 0}} a_{f}(A) \mathbf{e}(\operatorname{tr}(A Z)),
$$

be the Fourier expansion of $f(Z)$, where tr denotes the trace of a matrix. We call $f(Z)$ a cusp form if $a_{f}(A)=0$ unless $A$ is positive-definite. We denote by $\mathfrak{S}_{k}\left(\Gamma^{(n)}\right)$ the submodule of $\mathfrak{R}_{k}\left(\Gamma^{(n)}\right)$ consisting of cusp forms. Let $d v$ denote the invariant volume element on $\mathbf{H}_{n}$ defined by $d v=\operatorname{det}(\operatorname{Im}(Z))^{-n-1} \wedge_{1 \leq j \leq l \leq n}$ 
$\left(d x_{j l} \wedge d y_{j l}\right)$. Here for $Z \in \mathbf{H}_{n}$ we write $Z=\left(x_{j l}\right)+\sqrt{-1}\left(y_{j l}\right)$ with real matrices $\left(x_{j l}\right)$ and $\left(y_{j l}\right)$. For two $C^{\infty}$-modular forms $f$ and $g$ of weight $k$ with respect to $\Gamma^{(n)}$ we define the Petersson scalar product $\langle f, g\rangle$ by

$$
<f, g>=\int_{\Gamma^{(n)} \backslash \mathbf{H}_{n}} f(Z) \overline{g(Z)} \operatorname{det}(\operatorname{Im}(Z))^{k} d v,
$$

provided the integral converges.

For a positive even integer $k$ we define the Siegel Eisenstein series $E_{n, k}(Z, s)$ of degree $n$ as

$E_{n, k}(Z, s)=\zeta(1-k-2 s) \prod_{i=1}^{[n / 2]} \zeta(1-2 k-4 s+2 i) \sum_{M \in \Gamma_{\infty}^{(n)} \backslash \Gamma^{(n)}} j(M, Z)^{-k}(\operatorname{det}(\operatorname{Im}(M(Z))))^{s}$

$\left(Z \in \mathbf{H}_{n}, s \in \mathbf{C}\right.$ ), where $\zeta(*)$ is Riemann's zeta function, and $\Gamma_{\infty}^{(n)}=$ $\left\{\left(\begin{array}{c}* * \\ O_{n} *\end{array}\right) \in \Gamma^{(n)}\right\}$. Then $E_{n, k}(Z, s)$ is holomorphic at $s=0$ as a function of s. Furtheremore, assume that $k \geq(n+1) / 2$. Then $E_{n, k}(Z, 0)$ is holomorphic as a function of $Z$ unless $k=(n+2) / 2 \equiv 2 \bmod 4$, or $k=(n+3) / 2 \equiv 2 \bmod 4$ (cf. [28]). From now on we assume that $E_{n, k}(Z, 0)$ is holomorphic as a function of $Z$, and write $E_{n, k}(Z)=E_{n, k}(Z, 0)$. To see the Fourier expansion of $E_{n, k}(Z, 0)$, for a half-integral matrix $B$ of degree $n$ over $\mathbf{Z}$, we define the Siegel series $b(B, s)$ by

$$
b(B, s)=\sum_{R \in S_{n}(\mathbf{Q}) / S_{n}(\mathbf{Z})} \mathbf{e}(\operatorname{tr}(B R)) \mu(R)^{-s},
$$

where $\mu(R)=\left[R \mathbf{Z}^{n}+\mathbf{Z}^{n}: \mathbf{Z}^{n}\right]$. Furthermore we put

$$
\Gamma_{n}(s)=\prod_{j=1}^{n} \pi^{j / 2} \Gamma(s-(j-1) / 2),
$$

where $\Gamma(s)$ is Gamma function. For a $p$-adic number $x$ put $\mathbf{e}_{p}(x)=\exp (2 \pi \sqrt{-1} \tilde{x})$, where $\tilde{x}$ denotes a rational number such that $\tilde{x}-x \in \mathbf{Z}_{p}$. To investigate the Siegel series, for a prime number $p$ and a half-integral matrix $B$ of degree $n$ over $\mathbf{Z}_{p}$ define the local Siegel series $b_{p}(B, s)$ by

$$
b_{p}(B, s)=\sum_{R \in S_{n}\left(\mathbf{Q}_{p}\right) / S_{n}\left(\mathbf{Z}_{p}\right)} \mathbf{e}_{p}(\operatorname{tr}(B R)) \mu_{p}(R)^{-s},
$$

where $\mu_{p}(R)=\left[R \mathbf{Z}_{p}^{n}+\mathbf{Z}_{p}^{n}: \mathbf{Z}_{p}^{n}\right]$. Then we easily see that for a half-integral matrix $B$ of degree $n$ over $\mathbf{Z}$ we have

$$
b(B, s)=\prod_{p} b_{p}(B, s) .
$$

For a half-integral matrix $B$ of even degree $n$ define $\xi_{p}(B)$ by

$$
\xi_{p}(B)=\chi_{p}\left((-1)^{n / 2} \operatorname{det} B\right) .
$$


Let $B \in \mathcal{H}_{n}(\mathbf{Z})_{>0}$ with $n$ even. Then we can write $(-1)^{n / 2} 2^{n} \operatorname{det} B=\mathfrak{b}_{B} \hat{f}_{B}^{2}$ with $\grave{D}_{B}$ a fundamental discriminant and $\mathfrak{i}_{B} \in \mathbf{Z}_{>0}$. Furthermore, let $\chi_{B}=$ $\left(\frac{\emptyset_{B}}{*}\right)$ be the Kronecker character corresponding to $\mathbf{Q}\left(\sqrt{(-1)^{n / 2} \operatorname{det} B}\right) / \mathbf{Q}$.

We note that we have $\chi_{B}(p)=\xi_{p}(B)$ for any prime $p$. Let $H_{k}=\overbrace{H \perp \ldots \perp H}^{k}$ with $H=\left(\begin{array}{cc}0 & 1 / 2 \\ 1 / 2 & 0\end{array}\right)$.

For a non-degenerate half-integral matrix $B$ of degree $n$ over $\mathbf{Z}_{p}$ define a polynomial $\gamma_{p}(B, X)$ in $X$ by

$$
\gamma_{p}(B, X)= \begin{cases}(1-X) \prod_{i=1}^{n / 2}\left(1-p^{2 i} X^{2}\right)\left(1-p^{n / 2} \xi_{p}(B) X\right)^{-1} & \text { if } n \text { is even } \\ (1-X) \prod_{i=1}^{n-1} / 2\left(1-p^{2 i} X^{2}\right) & \text { if } n \text { is odd }\end{cases}
$$

Then the following lemma is well known (e.g. [20], Lemma 1)

Lemma 2.1 For a non-degenerate half-integral matrix $B$ of degree $n$ over $\mathbf{Z}_{p}$ there exists a unique polynomial $F_{p}(B, X)$ in $X$ over $\mathbf{Z}$ with constant term 1 such that

$$
b_{p}(B, s)=\gamma_{p}\left(B, p^{-s}\right) F_{p}\left(B, p^{-s}\right) \text {. }
$$

Remark. For an element $B \in \mathcal{H}_{n}\left(\mathbf{Z}_{p}\right)$ of rank $m \geq 0$, there exists an element $\tilde{B} \in \mathcal{H}_{m}\left(\mathbf{Z}_{p}\right) \cap G L_{m}\left(\mathbf{Q}_{p}\right)$ such that $B \sim \tilde{B} \perp O_{n-m}$. We note that $b_{p}(\tilde{B}, s)$ does not depend on the choice of $\tilde{B}$ (cf. [20]). Thus we write this as $b_{p}^{(m)}(B, s)$. Furthermore, $F_{p}(\tilde{B}, X)$ does not depend on the choice of $\tilde{B}$. Then we put $F_{p}^{(m)}(B, X)=F_{p}(\tilde{B}, X)$. For an element $B \in \mathcal{H}_{n}(\mathbf{Z})_{\geq 0}$ of rank $m \geq 0$, there exist an element $\tilde{B} \in \mathcal{H}_{m}(\mathbf{Z})_{>0}$ such that $B \sim \tilde{B} \perp O_{n-m}$. Then by the above remark $b(\tilde{B}, s)$ does not depend on the choice of $\tilde{B}$. Thus we write this as $b^{(m)}(B, s)$. Furthermore, $\operatorname{det} \tilde{B}$ does not depend on the choice of $B$. Thus we put $\operatorname{det}^{(m)} B=\operatorname{det} \tilde{B}$. Similarly, we write $\chi_{B}^{(m)}=\chi_{\tilde{B}}$ if $m$ is even.

Now for a semi-positive definite half-integral matrix $B$ of degree $2 n$ and of rank $m$, we put

$$
\begin{gathered}
c_{2 n, l}(B)=2^{[(m+1) / 2]} \prod_{p} F_{p}^{(m)}\left(B, p^{l-m-1}\right) \\
\times\left\{\begin{array}{ll}
\prod_{i=m / 2+1}^{n} \zeta(1+2 i-2 l) L\left(1+m / 2-l, \chi_{B}^{(m)}\right) & \text { if } m \text { is even } \\
(-1)^{\left(m^{2}-1\right) / 8} \prod_{i=(m+1) / 2}^{n} \zeta(1+2 i-2 l) & \text { if } m \text { is odd }
\end{array} .\right.
\end{gathered}
$$

Here we make the convention $F_{p}^{(m)}\left(B, p^{l-m-1}\right)=1$ and $L\left(1+m / 2-l, \chi_{B}^{(m)}\right)=$ $\zeta(1-l)$ if $m=0$. Then we have 
Theorem 2.2. Let $l$ be a positive even integer. Assume that $l \geq n+3$ or $l \geq n+1$ according as $n \equiv 1 \bmod 4$ or not. Then we have

$$
E_{2 n, l}(Z)=\sum_{B \in \mathcal{H}_{2 n}(\mathbf{Z}) \geq 0} c_{2 n, l}(B) \mathbf{e}(\operatorname{tr}(B Z)) .
$$

Remark. $c_{2 n, l}(B)$ is a rational number, and any prime divisor of its denominator is not greater than $(2 l-1)$ !. This is a weaker version of Böcherer's result in [4].

\section{Differential operators}

In this section, following [5], [16], we introduce some differential operators acting on the space of modular forms. Let $X=\left(x_{i j}\right)_{1 \leq i \leq m, 1 \leq j \leq d}$ be a matrix of variables, and for $1 \leq i, j \leq m$, put $\Delta_{i, j}=\sum_{\nu=1}^{d} \frac{\partial^{2}}{\partial x_{i \nu} \partial x_{j \nu}}$. A polynomial $P(X)$ in $X$ is called pluriharmonic if $\Delta_{i j} P=0$ for any $1 \leq i, j, \leq m$. Take a polynomial mapping $P\left(X_{1}, X_{2}\right)$ from $M_{n, 2 l}(\mathbf{C}) \times M_{n, 2 l}(\mathbf{C})$ to $\mathbf{C}$ such that D-1. $P\left(X_{1}, X_{2}\right)$ is pluriharmonic for each $X_{i}(i=1,2)$.

D-2. $P\left(X_{1} g, X_{2} g\right)=P\left(X_{1}, X_{2}\right)$ for any $g \in O(2 l)$, where $O(2 l)$ is the orthogonal group of degree $2 l$.

D-3. $P\left(a_{1} X_{1}, a_{2} X_{2}\right)=\left(\operatorname{det} a_{1}\right)^{\nu}\left(\operatorname{det} a_{2}\right)^{\nu} P\left(X_{1}, X_{2}\right)$ for $a_{1}, a_{2} \in G L_{n}(\mathbf{C})$.

Assume that $l \geq n$. Then there exists a unique polynomial mapping $Q(W)$ from $S_{2 n}(\mathbf{C})$ to $\mathbf{C}$ s.t $P\left(X_{1}, X_{2}\right)=Q\left(\left(\begin{array}{c}X_{1}{ }^{t} X_{1} X_{1}{ }^{t} X_{2} \\ X_{2}{ }^{t} X_{1} X_{2}{ }^{t} X_{2}\end{array}\right)\right)$. We note that $\operatorname{deg} Q=n \nu$. Let $Z=\left(z_{i j}\right)_{1 \leq i, j \leq 2 n}$ be a matrix of variables with $z_{i j}=z_{j i}$, and we write $\frac{\tilde{\partial}}{\partial z_{i j}}=\frac{\left(1+\delta_{i, j}\right)}{2} \frac{\partial}{\partial z_{i j}}$, and $\left(\frac{\partial}{\partial Z}\right)=\left(\frac{\tilde{\partial}}{\partial z_{i j}}\right)_{1 \leq i, j \leq 2 n}$. For $f \in C^{\infty}\left(\mathbf{H}_{2 n}\right)$ we define $\mathcal{D}_{Q}(f)$ and $\tilde{\mathcal{D}}_{Q}(f)$ by

$$
\mathcal{D}_{Q}(f)=Q\left(\frac{\partial}{\partial Z}\right)(f)
$$

and

$$
\tilde{\mathcal{D}}_{Q}(f)=\mathcal{D}_{Q}(f)_{Z_{12}=0}
$$

where we write $Z=\left(\begin{array}{cc}Z_{1} & Z_{12} \\ { }^{t} Z_{12} & Z_{2}\end{array}\right)$ with $Z_{1}, Z_{2} \in \mathbf{H}_{n}$ and $Z_{12} \in M_{n}(\mathbf{C})$. We consider the action of the above operators on the Fourier series. Let $A=\left(a_{i j}\right) \in \mathcal{H}_{2 n}$. Then we have $\mathrm{e}(\operatorname{tr}(A Z))=\exp \left(2 \pi \sqrt{-1}\left(\sum_{\alpha, \beta=1}^{2 n} a_{\alpha \beta} z_{\alpha \beta}\right)\right)$.

Then we have

$$
\frac{\tilde{\partial}}{\partial_{\alpha \beta}}(\mathbf{e}(\operatorname{tr}(A Z)))=2 \pi \sqrt{-1} a_{\alpha \beta} \mathbf{e}(\operatorname{tr}(A Z))
$$

Thus we have

$$
\mathcal{D}_{Q}(\mathbf{e}(\operatorname{tr}(A Z)))=(2 \pi \sqrt{-1})^{n \nu} Q(A) \mathbf{e}(\operatorname{tr}(A Z)) .
$$


Now let $Z=\left(\begin{array}{cc}Z_{1} & Z_{12} \\ { }^{t} Z_{12} & Z_{2}\end{array}\right) \in \mathbf{H}_{2 n}$ as above, and $f(Z)=\sum_{A \in \mathcal{H}_{2 n}(\mathbf{Z})_{\geq 0}} a(A) \mathbf{e}(\operatorname{tr}(A Z))$.

Then we have

$$
\tilde{\mathcal{D}}_{Q}(f)\left(Z_{1}, Z_{2}\right)
$$

$=(2 \pi \sqrt{-1})^{n \nu} \sum_{A_{1}, A_{2} \in \mathcal{H}_{n}(\mathbf{Z}) \geq 0} \mathrm{e}\left(\operatorname{tr}\left(A_{1} Z_{1}+A_{2} Z_{2}\right)\right) \sum_{R \in M_{n}(\mathbf{Z})} Q\left(\left(\begin{array}{cc}A_{1} & \frac{1}{2} R \\ \frac{1}{2}^{t} R & A_{2}\end{array}\right)\right) a\left(\left(\begin{array}{cc}A_{1} & \frac{1}{2} R \\ \frac{1}{2} t^{t} R & A_{2}\end{array}\right)\right)$.

For $\gamma=\left(\begin{array}{ll}a & b \\ c & d\end{array}\right) \in G S p_{n}(\mathbf{R})^{+}$put $\gamma^{\uparrow}=\left(\begin{array}{llll}a & 0 & b & 0 \\ 0 & 1 & 0 & 0 \\ c & 0 & d & 0 \\ 0 & 0 & 0 & 1\end{array}\right)$ and $\gamma^{\downarrow}=\left(\begin{array}{llll}1 & 0 & 0 & 0 \\ 0 & a & 0 & b \\ 0 & 0 & 1 & 0 \\ 0 & c & 0 & d\end{array}\right)$.

We define the mapping $\iota$ from $S p_{n}(\mathbf{R}) \times S p_{n}(\mathbf{R})$ to $S p_{2 n}(\mathbf{R})$ by

$$
\iota: S p_{n}(\mathbf{R}) \times S p_{n}(\mathbf{R}) \ni\left(\gamma_{1}, \gamma_{2}\right) \hookrightarrow \gamma_{1}^{\uparrow} \gamma_{2}^{\downarrow} \in S p_{2 n}(\mathbf{R})
$$

Furthermore, for a function $f: \mathbf{H}_{n} \times \mathbf{H}_{n} \longrightarrow \mathbf{C}, \gamma_{1}, \gamma_{2} \in S p_{n}(\mathbf{R})$ we define

$$
\left.f\right|_{l}\left(\gamma_{1}, \gamma_{2}\right)\left(Z_{1}, Z_{2}\right)=j\left(\gamma_{1}, Z_{1}\right)^{-l} j\left(\gamma_{2}, Z_{2}\right)^{-l} f\left(\gamma_{1}\left(Z_{1}\right), \gamma_{2}\left(Z_{2}\right)\right) .
$$

Then we have

Theorem 3.1. ([16])

$$
\left.\tilde{\mathcal{D}}_{Q}(f)\right|_{l+\nu}\left(\gamma_{1}, \gamma_{2}\right)=\tilde{\mathcal{D}}_{Q}\left(\left.f\right|_{l} \iota\left(\gamma_{1}, \gamma_{2}\right)\right)
$$

Now we apply the above theorem to the modular forms. For a subspace $\mathfrak{M}$ of $\mathfrak{M}_{l}^{\infty}\left(\Gamma^{(n)}\right)$ let $\mathfrak{R} \otimes \mathfrak{M}=\left\{\sum_{i, j} a_{i j} f_{i}\left(Z_{1}\right) f_{j}\left(Z_{2}\right)\right.$ (finite sum); $f_{i}, f_{j} \in \mathfrak{M}, a_{i j} \in$ C . Put $C_{q}(s)=s(s+1 / 2) \cdots(s+(q-1) / 2)$. We choose $Q=Q_{n, l}^{\nu}$ such that

$$
\tilde{\mathcal{D}}_{Q_{n, l}^{\nu}}\left(\operatorname{det} Z_{12}^{\nu}\right)=(-1)^{n \nu} \prod_{\mu=1}^{\nu}\left(C_{n}(\mu / 2) C_{n}(l-n+\nu-\mu / 2)\right)
$$

and put

$$
\stackrel{\circ}{\mathcal{D}}_{n, l}^{\nu}=\tilde{\mathcal{D}}_{Q_{n, l}^{\nu}}
$$

This coincides with $\stackrel{\circ}{D}_{n, l}^{\nu}$ in [5]. Then by Theorem 3.1 we easily see

Theorem 3.2. $\stackrel{\circ}{\mathcal{D}}_{n, l}^{\nu}$ maps $\mathfrak{M}_{l}^{\infty}\left(\Gamma^{(2 n)}\right)$ to $\mathfrak{M}_{l+\nu}^{\infty}\left(\Gamma^{(n)}\right) \otimes \mathfrak{M}_{l+\nu}^{\infty}\left(\Gamma^{(n)}\right)$. Furthermore $\stackrel{\circ}{\mathcal{D}}_{n, l}^{\nu}$ maps $\mathfrak{M}_{l}\left(\Gamma^{(2 n)}\right)$ into $\mathfrak{M}_{l+\nu}\left(\Gamma^{(n)}\right) \otimes \mathfrak{M}_{l+\nu}\left(\Gamma^{(n)}\right)$, and in particular if $\nu>0$, its image is contained in $\Theta_{l+\nu}\left(\Gamma^{(n)}\right) \otimes \bigotimes_{l+\nu}\left(\Gamma^{(n)}\right)$. 


\section{Pullback formula}

Let $\mathbf{L}_{n}=\mathbf{L}_{\mathbf{Q}}\left(G S p_{n}(\mathbf{Q})^{+}, \Gamma^{(n)}\right)$ denote the Hecke ring over $\mathbf{Q}$ associated with the Hecke pair $\left(G S p_{n}(\mathbf{Q})^{+}, \Gamma^{(n)}\right)$. For each integer $m$ define an element $T(m)$ of $\mathbf{L}_{n}$ by

$$
T(m)=\sum_{d_{1}, \ldots, d_{n}, e_{1}, \ldots, e_{n}} \Gamma^{(n)}\left(d_{1} \perp \ldots \perp d_{n} \perp e_{1} \perp \ldots \perp e_{n}\right) \Gamma^{(n)},
$$

where $d_{1}, \ldots, d_{n}, e_{1}, \ldots, e_{n}$ run over all positive integer satisfying

$$
d_{i}\left|d_{i+1}, e_{i+1}\right| e_{i}(i=1, \ldots, n-1), d_{n} \mid e_{n}, d_{i} e_{i}=m(i=1, \ldots, n) .
$$

Furthermore, for $i=0,1, \ldots, n$ and a prime number $p$, put

$$
T_{i}\left(p^{2}\right)=\Gamma^{(n)}\left(1_{n-i} \perp p 1_{i} \perp p^{2} 1_{n-i} \perp p 1_{i}\right) \Gamma^{(n)} .
$$

As is well known, $\mathbf{L}_{n}$ is generated over $\mathbf{Q}$ by all $T(p)$ and $T_{i}\left(p^{2}\right)(i=1, \ldots, n)$. We denote by $\mathbf{L}_{n}^{\prime}$ the subalgebra of $\mathbf{L}_{n}$ generated by over $\mathbf{Z}$ by all $T(p)$ and $T_{i}\left(p^{2}\right)(i=1, \ldots, n)$. Let $T=\Gamma^{(n)} M \Gamma^{(n)}$ be an element of $\mathbf{L}_{n} \otimes \mathbf{C}$. Write $T$ as $T=\cup_{\gamma} \Gamma^{(n)} \gamma$ and for $f \in \mathfrak{M}_{k}\left(\Gamma^{(n)}\right)$ define the Hecke operator $\left.\right|_{k} T$ associated to $T$ as

$$
\left.f\right|_{k} T=\left.\operatorname{det}(M)^{k / 2-(n+1) / 2} \sum_{\gamma} f\right|_{k} \gamma .
$$

We call this action the Hecke operator as usual (cf. [1].) If $f$ is an eigenfunction of a Hecke operator $T \in \mathbf{L}_{n} \otimes \mathbf{C}$, we denote by $\lambda_{f}(T)$ its eigenvalue. We call $f \in \mathfrak{M}_{k}\left(\Gamma^{(n)}\right)$ a Hecke eigenform if it is a common eigenfunction of all Hecke operators. Furthermore, we denote by $\mathbf{Q}(f)$ the field generated over $\mathbf{Q}$ by eigenvalues of all $T \in \mathbf{L}_{n}$ as in Section 1 . As is well known, $\mathbf{Q}(f)$ is a totally real algebraic number field of finite degree. Now, first we consider the integrality of the eigenvalues of Hecke operators. For an algebraic number field $K$, let $\mathfrak{D}_{K}$ denote the ring of integers in $K$.

Theorem 4.1. Let $k \geq n+1$. Let $f \in \mathfrak{S}_{k}\left(\Gamma^{(n)}\right)$ be a common eigenfunction of all Hecke operators in $\mathbf{L}_{n}^{\prime}$. Then $\lambda_{f}(T)$ belongs to $\mathfrak{D}_{\mathbf{Q}(f)}$ for any $T \in \mathbf{L}_{n}^{\prime}$.

The above theorem is known in case $n=1,2$ (cf. [24]), and it seems more or less well known also for general $n$. But for the readers' convenience, we here give a proof to it. Let $R$ be a subring of $\mathbf{C}$. Let $\mathfrak{S}_{k}\left(\Gamma^{(n)}\right)(R)$ be the $\mathbf{Z}$ module consisting of elements of $\mathfrak{S}_{k}\left(\Gamma^{(n)}\right)$ whose Fourier coefficients belong to $R$. It is known that we have $\mathfrak{S}_{k}\left(\Gamma^{(n)}\right)(\mathbf{Z}) \otimes \mathbf{C}=\mathfrak{S}_{k}\left(\Gamma^{(n)}\right)$ (cf. Shimura [27]). Then Theorem 4.1 follows from the following proposition:

Proposition 4.2. Assume that $k \geq n+1$. Let $R$ be a subring of $\mathbf{C}$. Any $T \in \mathbf{L}_{n}^{\prime}$ maps $\mathfrak{\Im}_{k}\left(\Gamma^{(n)}\right)(R)$ to itself.

Proof. Let

$$
f(z)=\sum_{A>0} a(A) \mathbf{e}(\operatorname{tr}(A z))
$$


be an element of $\mathfrak{S}_{k}\left(\Gamma^{(n)}\right)(R)$. Then we have $a(A) \in R$ for any $A$. For a element $T \in \mathbf{L}_{n}^{\prime}$ put

$$
f\left|T(z)=\sum_{A>0} a\right| T(A) \mathbf{e}(\operatorname{tr}(A z))
$$

Let $p$ be a prime number. First let $T=T(p)$. Then we have

$$
\begin{gathered}
a \mid T(p)(A)=p^{n k-n(n+1) / 2} \\
\left.\sum_{d_{1}\left|d_{2}\right| \cdots\left|d_{n}\right| p} \sum_{D \in G_{n} \backslash G_{n}\left(d_{1} \perp d_{2} \perp \cdots d_{n}\right) G_{n}}(\operatorname{det} D)^{-k} a\left(p^{-1} A^{t} D\right]\right),
\end{gathered}
$$

where $G_{n}=G L_{n}(\mathbf{Z})$ (e.g. Exercise 4.2 .10 of Andrianov [1].) Thus $a \mid T(p)(A)$ belongs to $R$ for any $A$. Next for a nonnegative integer $m$ and an integer $l$ put

$$
\beta_{p}(m, l)= \begin{cases}\prod_{i=1}^{l} \frac{p^{m-l+i}-1}{p^{i}-1} & \text { if } l \geq 1 \\ 1 & \text { if } l=0 \\ 0 & \text { if } l<0\end{cases}
$$

For $j=0,1, \ldots, n$ put

$$
\hat{T}_{j}\left(p^{2}\right)=\sum_{t=0}^{j} \beta_{p}(n-t, j-t) T_{t}\left(p^{2}\right),
$$

where $T_{0}\left(p^{2}\right)=\hat{T}_{0}\left(p^{2}\right)=\Gamma^{(n)} 1_{2 n} \Gamma^{(n)}$. The matrix $\left(\beta_{p}(n-t+1, j-t)\right)_{1 \leq j, t \leq n+1}$ is unimodular. Thus, to complete the proof, it suffices to show that $a \mid \hat{T}_{j}\left(p^{2}\right)(A) \in$ $R$ for any $0 \leq j \leq n$ and $A>0$. Now for a non-negative integers $r_{0}, r_{2}$ such that $r_{0}+r_{2} \leq n$, put

$$
\begin{gathered}
E\left(j, r_{0}, r_{2}\right)=k j-j(n+1)+k\left(r_{2}-r_{0}\right)+r_{0}\left(n-r_{2}+1\right)+\left(j-r_{0}-r_{2}\right)\left(j-r_{0}-r_{2}+1\right) / 2, \\
G_{n, r_{0}, r_{2}}=G_{n} \cap\left(\begin{array}{ccc}
\mathbf{Z}^{r_{0}, r_{0}} & \mathbf{Z}^{r_{0}, n-r_{0}-r_{2}} & \mathbf{Z}^{r_{0}, r_{2}} \\
p \mathbf{Z}^{n-r_{2}-r_{0}, r_{0}} & \mathbf{Z}^{n-r_{2}-r_{0}, n-r_{0}-r_{2}} & \mathbf{Z}^{n-r_{2}-r_{0}, r_{2}} \\
p^{2} \mathbf{Z}^{r_{2}, r_{0}} & p \mathbf{Z}^{r_{2}, n-r_{0}-r_{2}} & \mathbf{Z}^{r_{2}, r_{2}}
\end{array}\right),
\end{gathered}
$$

and

$$
D_{r_{0}, r_{2}}=p^{-1} \mathbf{1}_{r_{0}} \perp 1_{n-r_{0}-r_{2}} \perp p 1_{r_{2}} .
$$

Then, by Theorem 4.1 of Hafner and Walling [15], we have

$$
=p^{n(k-n-1)} \sum_{\substack{0 \leq r_{0}, r_{2} \leq n \\ r_{0}+r_{2} \leq j}} p^{E\left(j, r_{0}, r_{2}\right)} \sum_{D \in G_{n} / G_{n, r_{0}, r_{2}}}^{a \mid \hat{T}_{j}\left(p^{2}\right)(A)} \alpha\left(A, A\left[G D_{r_{0}, r_{2}}^{-1}\right]\right) a\left(A\left[G D_{r_{0}, r_{2}}^{-1}\right]\right),
$$

where $\alpha\left(A, A\left[G D_{r_{0}, r_{2}}^{-1}\right]\right)$ is a certain integer determined by $A$ and $A\left[G D_{r_{0}, r_{2}}^{-1}\right]$. (We note that the action of Hecke operators on the modular forms in their paper [15] is slightly different from ours. But the above statement is essentially 
the same as theirs.) Thus, $a \mid \hat{T}_{j}\left(p^{2}\right)(A)$ belongs to $R$, and we complete the proof.

Put

$$
G S p_{n}\left(\mathbf{Q}_{p}\right)=\left\{M \in G L_{2 n}\left(\mathbf{Q}_{p}\right) ; J_{n}[M]=\kappa(M) J_{n} \text { with some } \kappa(M) \in \mathbf{Q}_{p}^{\times}\right\}
$$

and let $\mathbf{L}_{n p}=\mathbf{L}\left(G S p_{n}\left(\mathbf{Q}_{p}\right), G S p_{n}\left(\mathbf{Q}_{p}\right) \cap G L_{2 n}\left(\mathbf{Z}_{p}\right)\right)$ be the Hecke algebra associated with the pair $\left(G S p_{n}\left(\mathbf{Q}_{p}\right), G S p_{n}\left(\mathbf{Q}_{p}\right) \cap G L_{2 n}\left(\mathbf{Z}_{p}\right)\right)$. Now assume that $f$ is a common eigenfunction of all Hecke operators, and for each prime number $p$, let $\alpha_{0}(p), \alpha_{1}(p), \ldots, \alpha_{n}(p)$ be the Satake parameters of $\mathbf{L}_{n p}$ determined by $f$. We then define the standard zeta function $L(f, s, \underline{\mathrm{St}})$ by

$$
L(f, s, \underline{\mathrm{St}})=\prod_{p} \prod_{i=1}^{n}\left\{\left(1-p^{-s}\right)\left(1-\alpha_{i}(p) p^{-s}\right)\left(1-\alpha_{i}(p)^{-1} p^{-s}\right)\right\}^{-1} .
$$

Let $n$ and $l$ be integers satisfying the conditions in Theorem 2.2 , and $E_{2 n, l}(Z)=$ $E_{2 n, l}(Z, 0)$ the Eisenstein series in Section 2. We then define $\mathcal{E}_{2 n, l, k}\left(z_{1}, z_{2}\right)$ as

$$
\mathcal{E}_{2 n, l, k}\left(z_{1}, z_{2}\right)=(-1)^{l / 2+1} 2^{-n}(2 \pi \sqrt{-1})^{l-k}(l-n) \stackrel{\circ}{\mathcal{D}_{n, l}^{k-l}}\left(E_{2 n, l}\right)\left(z_{1}, z_{2}\right),
$$

where $z_{1}, z_{2} \in \mathbf{H}_{n}$. Let $f(z)=\sum_{A \in \mathcal{H}_{n}(\mathbf{Z})_{>0}} a(A) \mathbf{e}(\operatorname{tr}(A z))$ be a Hecke eigenform in $\mathfrak{S}_{k}\left(\Gamma^{(n)}\right)$. For a positive integer $m \leq k-n$ such that $m \equiv n \bmod 2$ put

$$
\begin{gathered}
A(f, m, \underline{\mathrm{St}})=(-1)^{n(m+1) / 2+1} 2^{-4 k n+3 n^{2}+n+(n-1) m+2} \\
\times \Gamma(m+1) \prod_{i=1}^{n} \Gamma(2 k-n-i) \frac{L(f, m, \underline{\mathrm{St}})}{<f, f>\pi^{-n(n+1) / 2+n k+(n+1) m}} .
\end{gathered}
$$

We note that all the Fourier coefficients of $\mathcal{E}_{2 n, l, k}\left(z_{1}, z_{2}\right)$ are rational and any prime divisor of its denominator is not greater than $(2 l-1)$ !. Then the following result is due to [3] and [25]:

Theorem 4.3. Let $l, k$ and $n$ be a positive integers. Assume that $k$ and $l+n$ is even, and $3 \leq l \leq k-n$ or $1 \leq l \leq k-n$ accorrding as $n \equiv 1 \bmod 4$ or not. Let $f \in \mathcal{S}_{k}\left(\bar{\Gamma}^{(n)}\right)$ be a common eigenfunction of all Hecke operators in $\mathbf{L}_{n}$. Then we have

$$
\left.<f, \mathcal{E}_{2 n, l+n, k}(*,-\bar{z})\right)>=\Lambda(f, l, \underline{\mathrm{St}}) f(z) .
$$

For two semi-positive definite half-integral matrices $A_{1}, A_{2}$ of degree $n$, put

$$
\epsilon_{l, k}\left(A_{1}, A_{2}\right)=\sum_{A_{2}-\frac{1}{4} A_{1}^{-1}[R] \geq 0} \tilde{c}_{2 n, l}\left(\left(\begin{array}{cc}
A_{1} & R / 2 \\
{ }^{t} R / 2 & A_{2}
\end{array}\right)\right) Q_{n, l}^{(k-l)}\left(\left(\begin{array}{cc}
A_{1} & R / 2 \\
{ }^{t} R / 2 & A_{2}
\end{array}\right)\right)
$$


where $\tilde{c}_{2 n, l}(A)=(-1)^{l / 2+1} 2^{-n}(l-n) c_{2 n, l}(A)$ for $A \in \mathcal{H}_{2 n}(\mathbf{Z})_{\geq 0}$. Furthermore, for each semi-positive definite half-integral matrix $A_{1}$ put

$$
\mathcal{F}_{l, k ; A_{1}}\left(z_{2}\right)=\sum_{A_{2} \in \mathcal{H}_{n}(\mathbf{Z})_{\geq 0}} \epsilon_{l, k}\left(A_{1}, A_{2}\right) \mathbf{e}\left(\operatorname{tr}\left(A_{2} z_{2}\right)\right) .
$$

We note that $\mathcal{F}_{l, k ; A_{1}}\left(z_{2}\right)$ belongs to $\mathfrak{M}_{k}\left(\Gamma^{(n)}\right)$, and

$$
\mathcal{E}_{2 n, l, k}\left(z_{1}, z_{2}\right)=\sum_{A_{1} \in \mathcal{H}_{n}(\mathbf{Z}) \geq 0} \mathcal{F}_{l, k ; A_{1}}\left(z_{2}\right) \mathbf{e}\left(\operatorname{tr}\left(A_{1} z_{1}\right)\right) .
$$

In particular, if $l<k, \mathcal{F}_{l, k ; A_{1}}\left(z_{2}\right)$ belongs to $\mathfrak{S}_{k}\left(\Gamma^{(n)}\right)$, and

$$
\mathcal{E}_{2 n, l, k}\left(z_{1}, z_{2}\right)=\sum_{A_{1} \in \mathcal{H}_{n}(\mathbf{Z})>0} \mathcal{F}_{l, k ; A_{1}}\left(z_{2}\right) \mathbf{e}\left(\operatorname{tr}\left(A_{1} z_{1}\right)\right)
$$

Take an orthogonal basis $\left\{f_{i}\right\}_{i=1}^{d_{1}}$ of $\Theta_{k}\left(\Gamma^{(n)}\right)$ consisting of Hecke eigenforms. Write

$$
f_{i}(z)=\sum_{A \in \mathcal{H}_{n}(\mathbf{Z})>0} a_{i}(A) \mathbf{e}(\operatorname{tr}(A z)) .
$$

Now we compute the value $A(f, l, \underline{\mathrm{St}})$.

Theorem 4.4. In addition to the above notation and the assumption, assume that $l \leq k-n-2$. Then for any positive definite half-integral matrix $A_{1}$ of degree $n$ we have

$$
\mathcal{F}_{l+n, k ; A_{1}}(z)=\sum_{i=1}^{d_{1}} \Lambda\left(f_{i}, l, \underline{\mathrm{St}}\right) a_{i}\left(A_{1}\right) \overline{f_{i}(-\bar{z})} .
$$

Remark. Since $\mathcal{F}_{k, k ; A_{1}}(z)$ is not a cusp form, the above formula does not hold for $l=k-n$. However, by modifying the above method, we can get a similar formula for this case.

\section{Congruence of modular forms}

In this section we consider the congruence between the Hecke eigenvalues of modular forms of the same weight. Let $K$ be an algebraic number filed, and $\mathfrak{D}=\mathfrak{D}_{K}$ the ring of integers in $K$. For a prime ideal $\mathfrak{P}$ of $\mathfrak{D}$, we denote by $\mathfrak{D}_{(\mathfrak{P})}$ the localization of $\mathfrak{D}$ at $\mathfrak{N}$ in $K$. Let $\mathfrak{A}$ be a fractional ideal in $K$. If $\mathfrak{A}=\mathfrak{P}^{e} \mathfrak{\mathfrak { B }}$ with $\mathfrak{B O}_{(\mathfrak{P})}=\mathfrak{D}_{(\mathfrak{P})}$ we write ord $\mathfrak{\mathfrak { y }}=e$. We simply write $\operatorname{ord}_{\mathfrak{N}}(c)=\operatorname{ord}_{\mathfrak{N}}((c))$ for $c \in K$. Then we have the following lemma. 
Lemma 5.1. Let $f_{1}, \ldots ., f_{d}$ be Hecke eigenforms in $\mathfrak{S}_{k}\left(\Gamma^{(n)}\right)$ linearly independent over $\mathbf{C}$, and $G$ an element of $\mathfrak{\Im}_{k}\left(\Gamma^{(n)}\right)$. Write

$$
f_{i}(z)=\sum_{A} a_{f_{i}}(A) \mathbf{e}(\operatorname{tr}(A z))
$$

for $i=1, \ldots, d$, and

$$
G(z)=\sum_{A} a_{G}(A) \mathbf{e}(\operatorname{tr}(A z))
$$

Let $K$ be the composite field of $\mathbf{Q}\left(f_{1}\right), \mathbf{Q}\left(f_{2}\right), \ldots$, and $\mathbf{Q}\left(f_{d}\right)$, and $\mathfrak{D}=\mathfrak{D}_{K}$. Let $\mathfrak{P}$ be a prime ideal of $\mathfrak{D}$. Assume that

(1) $a_{G}(A)$ belongs to $\mathfrak{D}_{(\mathfrak{P})}$ for any $A \in \mathcal{H}_{n}(\mathbf{Z})_{>0}$, and $a_{f_{1}}\left(A_{1}\right) \in \mathfrak{D}_{(\mathfrak{P})}^{*}$ for some $A_{1} \in \mathcal{H}_{n}(\mathbf{Z})_{>0}$.

(2) there exist $c_{1}, \ldots, c_{d} \in K$ such that $\operatorname{ord}_{\mathfrak{P}}\left(c_{1}\right)<0$ and

$$
G(z)=\sum_{i=1}^{d} c_{i} f_{i}(z) .
$$

Then there exists $i \neq 1$ such that we have

$$
\lambda_{f_{i}}(T) \equiv \lambda_{f_{1}}(T) \bmod \mathfrak{P}
$$

for any $T \in \mathbf{L}_{n}^{\prime}$.

Proof. The assertion for $d=2$ has been proved in [23], and the general case can easily be proved. But, for the readers' convenience, we here give a proof. By assumption, we have $d \geq 2$. We prove the assertion by the induction on $d$. First let $d=2$. Then we have

$$
G(z)=c_{1} f_{1}(z)+c_{2} f_{2}(z)
$$

and

$$
\left.G\right|_{k} T(z)=c_{1} \lambda_{f_{1}}(T) f_{1}(z)+c_{2} \lambda_{f_{2}}(T) f_{2}(z)
$$

for any $T \in \mathbf{L}_{n}^{\prime}$. Thus we have

$$
\left.G\right|_{k} T(z)-\lambda_{f_{2}}(T) G(z)=c_{1}\left(\lambda_{f_{1}}(T)-\lambda_{f_{2}}(T)\right) f_{1}(z) .
$$

In particular, we have

$$
a_{\left.G\right|_{k} T}\left(A_{1}\right)-\lambda_{f_{2}}(T) a_{G}\left(A_{1}\right)=c_{1}\left(\lambda_{f_{1}}(T)-\lambda_{f_{2}}(T)\right) a_{f_{1}}\left(A_{1}\right) .
$$

Thus by assumption, we have

$$
\operatorname{ord}_{\mathfrak{P}}\left(\lambda_{f_{1}}(T)-\lambda_{f_{2}}(T)\right) \geq \operatorname{ord}_{\mathfrak{P}}\left(c_{1}^{-1}\right)>0 .
$$

This proves the assertion for $d=2$. Let $d \geq 3$, and assume that the assertion holds for $d-1$. We have

$$
G(z)=\sum_{i=1}^{d} c_{i} f_{i}(z) .
$$


If we have $\lambda_{f_{1}}(T) \equiv \lambda_{f_{d}}(T) \bmod \mathfrak{P}$ for any $T \in \mathbf{L}_{n}^{\prime}$, the assertion holds. Otherwise, take $T \in \mathbf{L}_{n}^{\prime}$ such that $\lambda_{f_{1}}(T) \not \equiv \lambda_{f_{d}}(T) \bmod \mathfrak{p}$. Then we have

$$
\left.G\right|_{k} T(z)=\sum_{i=1}^{d} c_{i} \lambda_{f_{i}}(T) f_{i}(z)
$$

Thus we have

$$
\left.G\right|_{k} T(z)-\lambda_{f_{d}}(T) G(z)=\sum_{i=1}^{d-1} c_{i}\left(\lambda_{f_{i}}(T)-\lambda_{f_{d}}(T)\right) f_{i}(z) .
$$

All the Fourier coefficients of the left-hand side belong to $\mathfrak{D}_{(\mathfrak{P})}$, and $\operatorname{ord}_{\mathfrak{P}}\left(c_{1}\left(\lambda_{f_{1}}(T)-\right.\right.$ $\left.\left.\lambda_{f_{d}}(T)\right)\right)<0$. Thus by the induction hypothesis, there exists an integer $2 \leq$ $i \leq d-1$ satisfying the required condition.

Let $f$ be a Hecke eigenform in $\mathfrak{S}_{k}\left(\Gamma^{(n)}\right)$ and $M$ be a subspace of $\mathfrak{S}_{k}\left(\Gamma^{(n)}\right)$ stable under Hecke operators $T \in \mathbf{L}_{n}$. Assume that $M$ is contained in $(\mathbf{C} f)^{\perp}$, where $(\mathbf{C} f)^{\perp}$ is the orthogonal complement of $\mathbf{C} f$ in $\mathfrak{S}_{k}\left(\Gamma^{(n)}\right)$ with respect to the Petersson product. A prime ideal $\mathfrak{P}$ of $\mathfrak{D}_{\mathrm{Q}(f)}$ is called a congruence prime of $f$ with respect to $M$ if there exists a Hecke eigenform $g \in M$ such that

$$
\lambda_{f}(T) \equiv \lambda_{g}(T) \bmod \tilde{\mathfrak{P}}
$$

for any $T \in \mathbf{L}_{n}^{\prime}$, where $\tilde{\mathfrak{P}}$ is some prime ideal of $\mathfrak{D}_{\mathbf{Q}(f) \mathbf{Q}(g)}$ lying above $\mathfrak{P}$. If $M=(\mathbf{C} f)^{\perp}$, we simply call $\mathfrak{P}$ a congruence prime of $f$.

Now we consider the relation between the congruence primes and the standard zeta values. To consider this, we have to normalize the standard zeta value $A(f, l, \underline{\mathrm{St}})$ for a Hecke eigenform $f$ because it is not uniquely determined by the system of Hecke eigenvalues of $f$. We note that there is no reasonable normalization of cuspidal Hecke eigenform in the higher degree case unlike the elliptic modular case. Thus we define the following quantities: for a Hecke eigenform $f(z)=\sum_{A} a_{f}(A) \mathbf{e}(\operatorname{tr}(A z))$ in $\mathfrak{S}_{k}\left(\Gamma^{(n)}\right)$, let $\mathfrak{I}_{f}$ be the $\mathfrak{D}_{\mathbf{Q}(f)}$-module generated by all $a_{f}(A)^{\prime} s$. Then, by multiplying a suitable constant $c$ we may assume all $a_{f}(A)^{\prime} s$ are elements of $\mathbf{Q}(f)$ with bounded denominator. Then $\mathfrak{I}_{f}$ is a fractional ideal in $\mathbf{Q}(f)$, and therefore, so is $A(f, l, \underline{\mathrm{St}}) \mathfrak{S}_{f}^{2}$ if $l$ satisfies the condition in Theorem 4.3. We note that this fractional ideal does not depend on the choice of $c$. In particular, if we assume the multiplicity one property for the Hecke eigenforms, these values are uniquely determined by the system of eigenvalues of $f$. We also note that the value $N_{\mathbf{Q}(f)}(\Lambda(f, l, \underline{\mathrm{St}})) N\left(\mathfrak{S}_{f}\right)^{2}$ does not depend on the choice of $c$, where $N\left(\mathfrak{I}_{f}\right)$ is the norm of the ideal $\mathfrak{I}_{f}$. Then by Theorem 4.4 and Lemma 5.1 , we have

Theorem 5.2. Let $f$ be a Hecke eigenform in $\Theta_{k}\left(\Gamma^{(n)}\right)$. Let $l$ be a positive integer satisfying the condition in Theorem 4.4. Let $\mathfrak{P}$ be a prime ideal of $\mathfrak{D}$. Assume that $\operatorname{ord}_{\mathfrak{p}}\left(\Lambda(f, l, \underline{\mathrm{St}}) \mathfrak{I}_{f}^{2}\right)<0$ and that it does not divide $(2 l-1)$ !. Then $\mathfrak{P}$ is a congruence prime of $f$. In particular, if a rational prime number 
$p$ divides the denominator of $N_{\mathbf{Q}(f)}(\Lambda(f, l, \underline{\mathrm{St}})) N\left(\mathfrak{I}_{f}\right)^{2}$, then $p$ is divided by some congruence prime of $f$.

Now for a Hecke eigenform $f$ in $\mathfrak{S}_{k}\left(\Gamma^{(n)}\right)$, let $\mathfrak{z}_{f}$ denote the subspace of $\mathfrak{S}_{k}\left(\Gamma^{(n)}\right)$ spanned by all Hecke eigenforms with the same system of the Hecke eigenvalues as $f$.

Corollary. In addition to the above notation and the assumption, assume that $\Theta_{k}\left(\Gamma^{(n)}\right)$ has the multiplicity one property. Then $\mathfrak{\$}$ is a congruence prime of $f$ with respect to $\mathfrak{\Sigma}_{f}^{\perp}$. In particular, if a rational prime number $p$ divides the denominator of $N_{\mathbf{Q}(f)}(\Lambda(f, l, \underline{\mathrm{St}})) N\left(\mathfrak{I}_{f}\right)^{2}$, then $p$ is divided by some congruence prime of $f$ with respect to $\mathfrak{F}_{f}^{\perp}$

Remark. Let $f$ be an elliptic cuspidal Hecke eigenform. In [17] and [18], roughly speaking, we proved that prime divisors of the denominator of $L(f, m, \underline{\mathrm{St}}) /<f, f>$ are congruence primes of $f$, and Theorem 5.2 is a natural generalization of this result. On the other hand, in [13], Hida proved that prime divisors of the numerator of $L(f, 1, \underline{\mathrm{St}}) / \Omega^{+} \Omega^{-}$are congruence primes of $f$, where $\Omega^{+}$and $\Omega^{-}$are certain periods arising from the Eichler-Shimura cohomology. It seems difficult to generalize this result to Siegel modular forms of higher degree.

\section{A conjecture for the congruence of Saito-Kurokawa lift}

Let $J_{k, 1}^{\text {cusp }}$ be the space of Jacobi cusp forms of weight k and of index 1 on $\Gamma^{(1)}$, and $\mathcal{V}: J_{k, 1}^{\text {cusp }} \longrightarrow \mathfrak{S}_{k}\left(\Gamma^{(2)}\right)$ be the injection defined in Theorem 6.2 of Eichler and Zagier [9]. Then $\mathcal{V}\left(J_{k, 1}^{\text {cusp }}\right)$ is the Maass subspace of $\mathfrak{S}_{k}\left(\Gamma^{(2)}\right)$, which will be denoted by $\mathfrak{S}_{k}\left(\Gamma^{(2)}\right)^{*}$. Now we consider the congruence prime of the Saito-Kurokawa lift with respect to $\left(\Theta_{k}\left(\Gamma^{(2)}\right)^{*}\right)^{\perp}$. Let $\Theta_{k-1 / 2}\left(\Gamma_{0}(4)\right)^{+}$ be the Kohnen plus subspace in $\mathfrak{S}_{k-1 / 2}\left(\Gamma_{0}(4)\right)$. Then there exits an isomorphism $\mathcal{W}: \mathfrak{S}_{k-1 / 2}\left(\Gamma_{0}(4)\right)^{+} \longrightarrow J_{k, 1}^{\text {cusp }}$. For two modular forms $g, h$ in $\mathfrak{\Im}_{k-1 / 2}\left(\Gamma_{0}(4)\right)^{+}$, we define the Petersson product $\langle g, h\rangle$ as

$$
<g, h>=\frac{1}{6} \int_{\Gamma_{0}(4) \backslash \mathbf{H}_{1}} g(z) \overline{h(z)} y^{k-5 / 2} d x d y,
$$

where $z=x+\sqrt{-1} y$. Let $f(z)=\sum_{m=1}^{\infty} a_{f}(m) \mathbf{e}(m z)$ be a normalized Hecke eigenform in $\Theta_{2 k-2}\left(\Gamma^{(1)}\right)$. Let $\tilde{f}$ be an element of the Kohnen plus space $\mathfrak{S}_{k-1 / 2}\left(\Gamma_{0}(4)\right)^{+}$corresponding to $f$ via the Shimura correspondence. We note that $\tilde{f}$ is uniquely determined, up to constant multiple, by $f$. Put $\hat{f}=\mathcal{V} \circ$ $\mathcal{W}(\tilde{f})$, and call it the Saito-Kurokawa lift of $f$. We note that we have $\mathbf{Q}(\tilde{f})=$ $\mathbf{Q}(\hat{f})=\mathbf{Q}(f)$. Furthermore, we have $\mathfrak{I}_{\tilde{f}}=\mathfrak{I}_{\hat{f}}$, where $\mathfrak{I}_{\tilde{f}}$ is the $\mathfrak{D}_{\mathbf{Q}(f)}$-module generated by all the Fourier coefficients of $\tilde{f}$.

For a Dirichlet character $\chi$, let $L(f, s, \chi)$ be the Hecke L-function of $f$ twisted by $\chi$ define by as follows:

$$
L(f, s, \chi)=\sum_{m=1}^{\infty} a_{f}(m) \chi(m) m^{-s} .
$$


In particular, if $\chi$ is the principal character we write $L(f, s, \chi)$ as $L(f, s)$. Put $\Omega_{f}^{(+)}=(2 \pi \sqrt{-1})^{-1} L(f, 1)$, and $\Omega_{f}^{(-)}=(2 \pi \sqrt{-1})^{-2} L(f, 2)$. For $j= \pm, 1 \leq$ $l \leq 2 k-3$, and a Dirichlet character $\chi$ such that $\chi(-1)=j(-1)^{l-1}$, put

$$
\mathbf{L}(f, l, \chi)=\frac{(2 \pi \sqrt{-1})^{-l} \Gamma(l) L(f, l, \chi)}{\Omega^{(j)}} .
$$

In paricular, put $\mathbf{L}(f, l)=\mathbf{L}(f, l, \chi)$ if $\chi$ is the principal character. Furthermore, for positive integers $1 \leq m, m^{\prime} \leq 2 k-3$, put

$$
C\left(f, m, m^{\prime}\right)=\frac{L(f, m) L\left(f, m^{\prime}\right)}{(2 \pi \sqrt{-1})^{m+m^{\prime}}<f, f>} .
$$

Then it is well-known that $\mathbf{L}(f, l, \chi)$ belongs to the field $\mathbf{Q}(f)(\chi)$ generated over $\mathbf{Q}(f)$ by all the values of $\chi$, and that $C\left(f, m, m^{\prime}\right) \in \mathbf{Q}(f)$ if $m-m^{\prime}$ is odd (cf. Shimura [26].)

Let $\hat{f}$ be the Saito-Kurokawa lift of $f$. Then we have

$$
L(\hat{f}, s, \underline{\mathrm{St}})=\zeta(s) \prod_{i=1}^{2} L(f, s+k-i) .
$$

According to the numerical data in [19], we expect that a prime of the numerator of algebraic part of a certain L-value of $f$ is related to the congruence of $\hat{f}$. In fact, we would propose the following conjecture:

Conjecture. Let $\mathfrak{P}$ be a prime ideal of $\mathbf{Q}(f)$ not dividing $(2 k-1)$ !. Then $\mathfrak{P}$ is a congruence prime of $\hat{f}$ with respect to $\left(\Im_{k}\left(\Gamma^{(2)}\right)^{*}\right)^{\perp}$ if and only if $\mathfrak{P}$ divides the numerator of $\mathbf{L}(f, k)$.

Remark. The "if part" of the above conjecture is a special case of Harder's conjecture (cf. [11], [12] .) We also note that this is an analogue of the Doi-Hida-Ishii conjecture concerning the congruence primes of the Doi-Naganuma lifting [8].

Remark. We can also formulate the above conjecture by putting $\Omega_{f}^{-}=$ $\mathbf{L}(f, l)$ for an even positive integer $4 \leq l \leq k-4$. There might be another formulation of the conjecture in terms of the periods in Hida [14].

In this paper, we give some partial result for this conjecture.

Theorem 6.1. Let the notation be as above. Let $\mathfrak{P}$ be a prime ideal of $\mathbf{Q}(f)$ not dividing $(2 k-1)$ !. Assume that

(1) $\mathfrak{P}$ divides $\mathbf{L}(f, k)$

(2) $\mathfrak{P}$ is not a congruence prime of $f$

(3) does not divide $\mathbf{L}\left(f, k-1, \chi_{D}\right) D$ for some fundamental discriminant $D<0$, where $\chi_{D}$ is the Kronecker character corresponding to the extension $\mathbf{Q}(\sqrt{D}) / \mathbf{Q}$

(4) $\mathfrak{P}$ does not divide $C(f, 2 m+k-2,2 m+k-1) \zeta(1-2 m)$ for some integer $2 \leq m \leq k / 2-2$. 
Then $\mathfrak{P}$ is a congruence prime of $\hat{f}$ with respect to $\left(\bigodot_{k}\left(\Gamma^{(2)}\right)^{*}\right)^{\perp}$.

Remark. We expect that the above assertion holds without assuming the conditions (2),(3), and (4).

Proof. By the above consideration, for $2 \leq m \leq k / 2-2$ have

$$
\Lambda(\hat{f}, 2 m, \underline{\mathrm{St}})=C_{k, m} \frac{\zeta(1-2 m) L(f, 2 m+k-2) L(f, 2 m+k-1)}{\pi^{-3+2 k+4 m}<\hat{f}, \hat{f}>}
$$

where $C_{k, m}$ is a rational number whose numerator and denominator are not divisible by a prime number greater than $2 k-2$. Let $\tilde{f}$ be an element of the Kohnen plus space $\mathfrak{\subseteq}_{k-1 / 2}\left(\Gamma_{0}(4)\right)^{+}$corresponding to $f$ via the Shimura correspondence. Then by Kohnen and Skoruppa [21], we have

$$
\frac{\Gamma(k) L(f, k)}{(2 \pi)^{k}}=3 \cdot 2^{-k+3} \frac{<\hat{f}, \hat{f}>}{<\tilde{f}, \tilde{f}>} .
$$

We also have $\mathfrak{I}_{\hat{f}}=\mathfrak{I}_{\tilde{f}}$. On the other hand, by Kohnen and Zagier [22], we have

$$
\frac{c_{\tilde{f}}(|D|)^{2}}{<\tilde{f}, \tilde{f}>}=\frac{\Gamma(k-1)|D|^{k-3 / 2} L\left(f, k-1, \chi_{D}\right)}{\pi^{k-1}<f, f>} .
$$

By the assumption (3), we have

$$
\operatorname{ord}_{\mathfrak{P}}\left(\mathbf{L}\left(f, k-1, \chi_{D}\right)\right)=0 \text {. }
$$

Thus by the assumptions (1), we have

$$
\begin{gathered}
\operatorname{ord}_{\mathfrak{P}}\left(\Lambda(\hat{f}, 2 m, \underline{\mathrm{St}}) \mathfrak{I}_{\hat{f}}^{2}\right) \\
=\operatorname{ord}_{\mathfrak{P}}\left(C(f, 2 m+k-2, m+k-1) \zeta(1-2 m) \mathbf{L}(f, k)^{-1}\right)<0
\end{gathered}
$$

for some $2 \leq m \leq k / 2-2$. Thus by Theorem 5.2 and the assumption (2), we easily see that $\mathfrak{P}$ is a congruence prime of $\hat{f}$ with respect to $\left(\mathfrak{S}_{k}\left(\Gamma^{(2)}\right)^{*}\right)^{\perp}$. This completes the proof.

Remark. In [6], Brown has got a result similar to above.

Remark. We can formulate this type of conjecture for the congruence primes of the Ikeda lifting.

Acknowledgements The author thanks the refree for useful comments. 


\section{References}

1. A. N. Andrianov, Quadratic forms and Hecke operators, Springer, 1987.

2. S. Böcherer, Über die Fourier-Jacobi-Entwicklung Siegelscher Eisensteinreihen, Math. Z. 183(1983), 21-46.

3. S. Böcherer, Über die Fourier-Jacobi-Entwicklung Siegelscher Eisensteinreihen II, Math. Z. 189(1985), 81-110.

4. S. Böcherer, Über die Fourierkoeffizienten Siegelscher Eisensteinreihen, Manuscripta Math., 45(1984), 273-288.

5. S. Böcherer and C.G. Schmidt, p-adic measures attached to Siegel modular forms, Ann. Inst. Fourier, 50(2000), 1375-1443.

6. J. Brown, Saito-Kurokawa lifts and applications to the Bloch-Kato conjecture to appear in Compositio Math.

7. K. Doi and H. Hida, On certain congruence of cusp forms and the special values of their Dirichlet series, unpublished manuscript 1978.

8. K. Doi, H. Hida, and H. Ishii, Discriminant of Hecke fields and twisted adjoint L-values for GL(2), Invent. Math. 134(1998), 547-577.

9. M. Eichler and D. Zagier, The theory of Jacobi forms, Birkhäser, Boston, 1985.

10. P. Garrett, Pullbacks of Eisenstein series, Automorphic forms of several variables, Progress in Math. 46, Birkhäuser, 1984, 114-137.

11. G. Harder, Eisensteinkohomologie und die Konstruktion gemischter Motive, Lecture Notes. in Math. 1562, Springer-Verlag, Berlin 1993.

12. G. Harder, A congruence between a Siegel modular form and an elliptic modular form, Preprint 2003.

13. H. Hida, Congruences of cusp forms and special values of their zeta functions, Invent. Math. 64(1981), 221-262

14. H. Hida, Modular forms and Galois cohomology, Cambridge Univ. Press, 2000

15. J. L. Hafner and L. H. Walling, Explicit action of Hecke operators on Siegel modular forms, J. Number Theory, 93(2002), 34-57.

16. T. Ibukiyama, On Differential operators on automorphic forms and invariant pluri-harmonic polynomials, Comm. Math. Univ. St. Pauli, 48(1999), 103-118.

17. H. Katsurada, Special values of the zeta functions, Developments in Math $11(2003), 337-356$.

18. H. Katsurada, Special values of the standard zeta functions for elliptic modular forms, Experiment. Math. 14(2005), 27-45.

19. H. Katsurada, Exact values of the standard zeta functions for Sigel modular forms, Preprint.

20. Y. Kitaoka, Dirichlet series in the theory of quadratic forms, Nagoya Math. J. 92(1984), 73-84.

21. W. Kohnen and N-P. Skoruppa, A certain Dirichlet series attached to Siegel modular forms of degree 2, Invent. Math. 95, 541-558(1989).

22. W. Kohnen and D. Zagier, Values of L-series of modular forms at the center of the critical strip, Invent. Math. 64, 175-198(1981)

23. N. Kurokawa, Congruences between Siegel modular forms of degree two, Proc. Japan Acad. 57(1979) 417-422

24. N. Kurokawa, On Siegel eigenforms, Proc. Japan Acad. 57(1981), 47-50.

25. S. Mizumoto, Poles and residues of standard L-functions attached to Siegel modular forms, Math. Ann. 289(1991) 589-612.

26. G. Shimura, The special values of the zeta functions associated with cusp forms, Comm. pure appl. Math. 29(1976), 783-804.

27. G. Shimura, On the Fourier coefficients of modular forms of several variables, Göttingen, Nachr. Akad. Wiss. (1975), 261-268.

28. G. Shimura, Arithmeticity in the theory of automorphic forms, Mathematical Surveys and Monographs 82(2000), Amer. Math. Soc. 